\title{
Potenziale von PROFlenergy in der Prozessindustrie
}

\section{Einsatzpotenziale in der verfahrenstechnischen Optimierung}

\author{
Karl-Heinz Niemann, Andreas Würger, Hochschule Hannover
}

Der vorliegende Beitrag beschreibt Einsatzpotenziale des Energiemanagementprofils PROFlenergy in der Prozessindustrie. Der Blick auf den Status von Energieeffizienzmaßnahmen in der Prozessindustrie zeigt, dass diese im Wesentlichen innerhalb der verfahrenstechnischen Optimierung angesiedelt sind. Noch hat sich der durchgängige Einsatz von technischen Energiemanagementsystemen (tEnMS) nicht etabliert. Diese Arbeit fokussiert Vorteile des tEnMS-Einsatzes und präsentiert „Best Practice“Beispiele in der Prozessindustrie. Abschließend wird aufgezeigt, welches Potenzial das Energiemanagementprofil PROFlenergy liefern kann und welche Anwendungsfälle sich damit abdecken lassen.

\#Energiemanagement \#PROFlenergy \#Prozessindustrie \#Energieeffizienz

\section{Potentials of PROFlenergy in the process industry}

Application potentials in process engineering optimization

This article deals with the potential offered by the energy management profile PROFlenergy for the process industry. The examination of the status of energy efficiency measures in the process industry shows that efficiency measures occur mainly in the area of process optimization. The consistent use of technical energy management systems (tEnMS) is rather rare. The next step is to work out what advantages the use of a tEnMS brings and what can be regarded as "best practice" in the process industry. Finally, the article looks at the potential the PROFlenergy energy management profile can deliver in and which application cases can be covered by it.

\#energy management \#PROFlenergy \#process industry \#energy efficiency

Der Klimaschutz ist ein Thema von steigender politischer, aber auch gesellschaftlicher Relevanz sowohl in der Bundesrepublik Deutschland als auch darüber hinaus. So hat die Europäische Union das Ziel, die Treibhausgasemissionen im Jahr 2030 um 40 \% im Vergleich zum Basisjahr 1990 zu senken. Im selben Zeitraum soll der Anteil erneuerbarer Energien am Bruttoendenergieverbrauch auf $32 \%$ gesteigert werden. Außerdem soll die Energieeffizienz um 32,5\% gesteigert werden. [1] Energieeffizienz ist gemäß [2] definiert als ein möglichst hoher Nutzen (Bsp. Produkte, Dienstleistungen, etc.) bei möglichst geringem Energieeinsatz.

Betrachten wir den Stromverbrauch in der Bundesrepublik nach Sektoren, entfallen $45 \%$ auf den industriellen Sektor, $28 \%$ auf den Sektor Gewerbe, Handel und Dienstleistungen, $25 \%$ auf Privathaushalte und zwei Prozent auf den Verkehrssektor. Auf den Industriesektor entfällt also der mit Abstand größte Anteil des Stromverbrauchs in Deutschland. [3]

Abbildung 1 zeigt den Energieverbrauch der deutschen Industrie, aufgeteilt nach Wirtschaftszweigen. Die fünf Wirtschaftszweige mit den höchsten Energieverbräuchen sind der Prozessindustrie zuzuordnen. Damit ist auch zu erwarten, dass künftig entsprechend hohe Erwartungen hinsichtlich der Energieeinsparung auf diesen Industrien lasten werden, um die o.g. Klimaschutzziele zu erreichen. Die Einführung eines betrieblichen Energiemanagementsystems nach ISO 50001 [5] hilft Industrieunternehmen, Beschaffung, Wandlung, Verteilung und Nutzung von Energie kontinuierlich zu überwachen und zu optimieren. So können Energieverschwendungen aufgedeckt und Potenziale zur Erhöhung der Energieeffizienz gefunden werden. [6] Als Anreiz für die Einführung eines betrieblichen Energiemanagementsystems erhalten Unternehmen mit einer ISO-50001-Zertifizierung nach $\S 10$ StromStG steuerliche Vorteile.

Alle technischen Komponenten eines Energiemanagementsystems (Software, Hardware, Kommunikationssysteme, etc.) werden zusammen als technisches Energiemanagementsystem (im Folgenden wird hierfür die Abkürzung tEnMS verwendet) bezeichnet. Ein tEnMS dient überwiegend dem Messen, Speichern, Auswerten und Darstellen von Energiedaten, es kann jedoch auch über Funktionen zur gezielten Beeinflussung der Energieaufnahme von Verbrauchern verfügen. Derartige Funktionen werden als Lastmanagement [7] bezeichnet. 
Im Zusammenhang mit tEnMS gewinnen Energiemanagementprofile, wie z. B. PROFlenergy [8] für PROFINET oder Sercos Energy [9] für Sercos III, immer mehr an Bedeutung. Ein Energieprofil spezifiziert Methoden und Techniken zur Implementierung energiesparender Funktionen innerhalb eines IO-Devices. Dadurch verfügen die IO-Devices über standardisierte Energieschnittstellen, über welche diese z. B. Messwerte von integrierten Energiemessstellen bereitstellen können.

\section{Ausgangssituation in der Prozessindustrie}

Dieses Kapitel untersucht die aktuelle Situation hinsichtlich Energieeffizienz und Energiemanagement in der Prozessindustrie. Hierzu erfolgt zunächst eine Analyse relevanter Fachliteratur. Anschließend werden die Anforderungen der Betreiber von prozesstechnischen Anlagen betrachtet. Danach erfolgt eine Untersuchung der Potenziale für Lastmanagement in Anlagen der Prozessindustrie.

\subsection{Analyse relevanter Literatur}

In $[7,10,11]$ wird das Thema Energieeffizienz in der Prozessindustrie eingehend behandelt. Hieraus lassen sich sechs für die Prozessindustrie typische Wege für die Erhöhung der Energieeffizienz ableiten:

1. Minimierung von materiellen Abfällen und Optimierung des Prozesses.

2. Minimierung von Verlusten, z. B. durch Optimierung der Dampfverteilung.

3. Optimierung der Prozessfahrweise, bzw. -planung, z. B. durch optimierte Anpassung des Prozesses an die schwankende Qualität von Rohstoffen.

4. Rückgewinnung anfallender Wärme, bzw. Optimierung der Wärmerückgewinnung.

5. Veränderung von Prozessen in Richtung Energieeffizienz, z. B. durch Reduzierung von Temperaturen, Einsatz optimierter Wärmetauscher, Überwachung von Wärmetauschern, etc.

\section{Optimierung des Energieversorgungssystems.}

Des Weiteren leitet sich aus den Quellen ab, dass die Prozessindustrie die Energieeffizienz im Wesentlichen durch die Optimierung der verfahrenstechnischen Prozesse vorantreibt. Ein Energiemanagement, also die Einführung eines betrieblichen Energiemanagementsystems und der Einsatz eines tEnMS, steht oft im Hintergrund oder ist nicht vorgesehen [12]. Dabei existieren durchaus Potenziale für tEnMS in den Anlagen der Prozessindustrie. Vordergründig werden tEnMS eingesetzt, um eine durchgehende Energiedatenerfassung, für z. B. elektrische Energie, Prozesswärme (Dampf) und Prozesskälte, zu gewährleisten. Anhand der ermittelten Daten kann Energieverschwendung identifiziert und die Energieeffizienz somit erhöht werden.

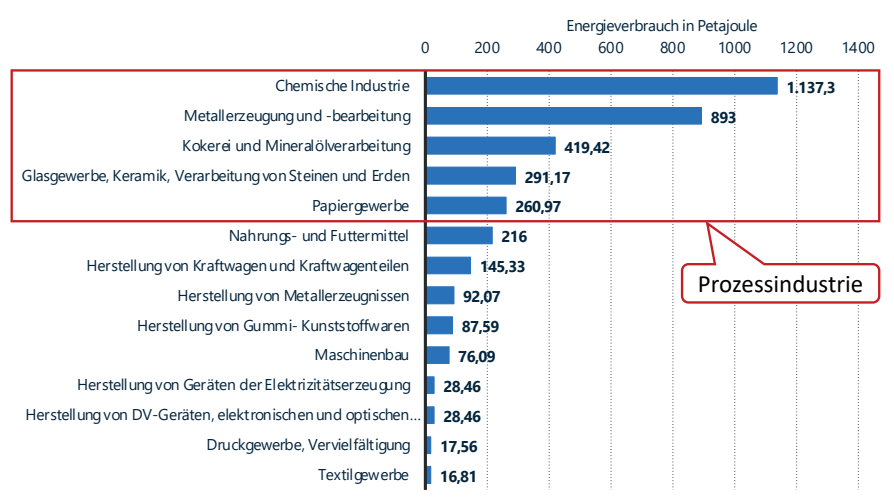

Abbildung 1: Energieverbrauch in der deutschen Industrie nach Wirtschaftszweigen [4].

\subsection{Anforderungen von Anlagenbetreibern}

Das NAMUR Arbeitsblatt NA 140 [13] beschreibt ein Vorgehen zur Steigerung der Energieeffizienz in der Prozessindustrie. Die NA 140 fordert unter anderem:

» Eine automatische und kontinuierliche Erfassung und Archivierung der Energieverbräuche,

» den Einsatz hinreichend genauer und betriebsbewährter Messsysteme,

» die Erfassung und Speicherung qualitätsrelevanter Zusatzdaten, wie z. B. elektrische Spannung, Druck, Durchfluss oder Temperatur,

» die Schaffung von Schnittstellen zu weiterführenden Auswertesystemen, wie z. B. dem Prozess-InformationsManagementsystem (PIMS), in welchem die Messstellen zu sinnvollen Gruppen zusammengefasst werden sowie

» die datenbasierte Auswertung einzelner Aggregate (z. B. Fouling von Wärmetauschern über die Überwachung von Wärmeübergangskoeffizienten).

Aus den aufgeführten Anforderungen lässt sich ableiten, dass den Betreibern prozesstechnischer Anlagen eine Energiedatenerfassung und -dokumentation empfohlen wird. Das NA 140 sieht die durchgängige Erfassung dieser Energiedaten als Basis einer kontinuierlichen, energetischen Überwachung des Betriebes. Diese dient zum einen zur Erkennung von Abweichungen (z. B. Verschlechterung der Energieeffizienz im laufenden Betrieb) und zum anderen als Basis zur Identifikation weiterer Einsparpotenziale. Durch eine detaillierte lokale Erfassung der Energiedaten kann auch eine entsprechende Kostenzuordnung erfolgen.

\subsection{Potenziale für das Lastmanagement}

Wie bereits in der Einleitung erwähnt, ist das Lastmanagement, also die gezielte Beeinflussung der Energieaufnahme von Verbrauchern, eine wesentliche Funktion von tEnMS. Der Beitrag [14] befasst sich mit Lastmanagement in der Industrie. Typische Anwendungsfälle eines Lastmanagements sind unter anderem: 


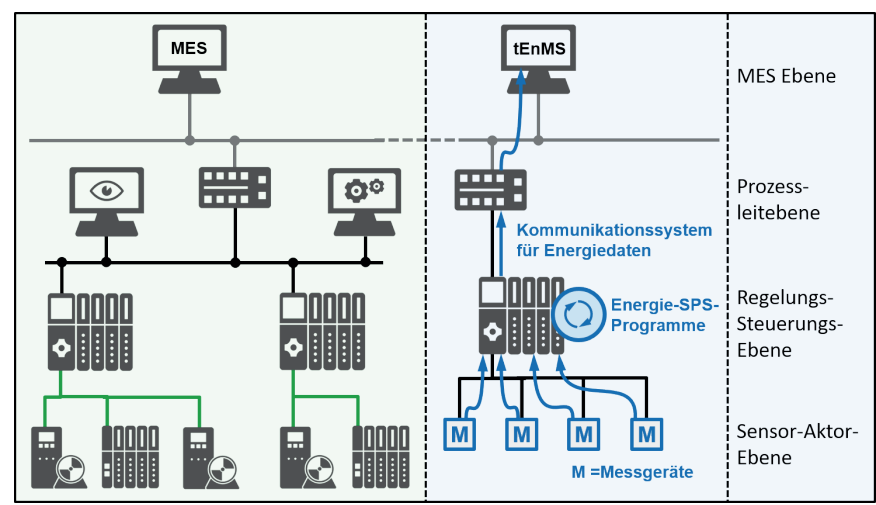

Abbildung 2: Parallel zur Automatisierungstechnik installiertes tEnMS, in Anlehnung an [17].

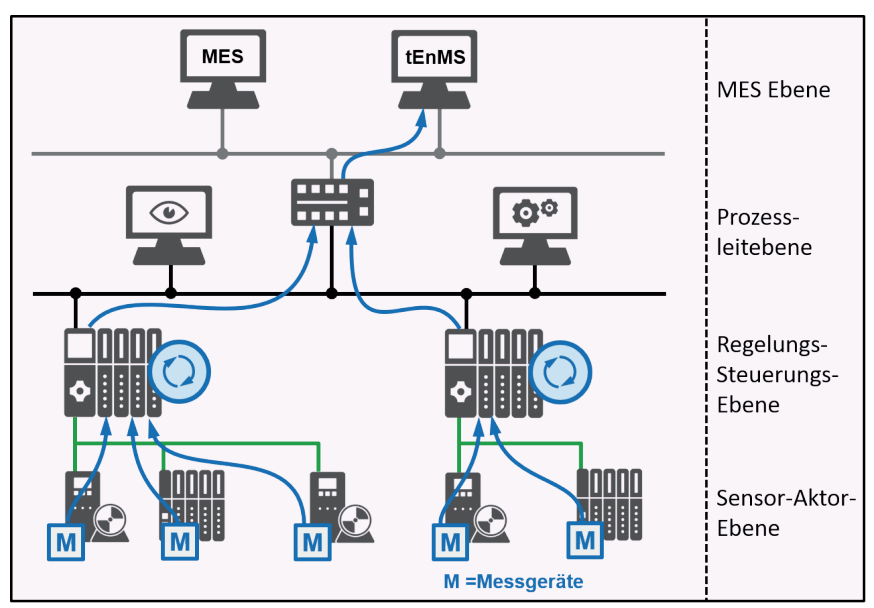

Abbildung 3: Integriertes tEnMS, in Anlehnung an [17].

a. Die Verstetigung des Energieverbrauchs zur Vermeidung von Lastspitzen.

b. Das gezielte Ab- oder Zuschalten von Verbrauchern zur Teilnahme am Regelenergiemarkt.

c. Das Energiesparen in geplanten Produktionspausen (z. B. bei Batch-Anlagen nachts oder am Wochenende) durch das Schalten von Verbrauchern in Energiesparmodi auch Standby-Management genannt.

In [14] werden unter anderem Verbraucher hinsichtlich ihrer Eignung auf Lastmanagement untersucht. Hieraus lässt sich folgendes ableiten: Verbraucher in prozesstechnischen Anlagen laufen oft über Zeiträume von Monaten oder gar Jahren ohne Pause durch. Sie können in der Regel nicht abgeschaltet werden. Die Anwendungsfälle b. und c. sind also in kontinuierlich arbeitenden, prozesstechnischen Anlagen wenig relevant, bzw. nicht realisierbar und bei Batch-Anlagen eingeschränkt realisierbar. Allerdings existieren in prozesstechnischen Anlagen auch Verbraucher, die sich den sogenannten Infrastrukturtechnologien zuordnen lassen, wie z. B. Lüfter oder Wärme- und Kälteerzeuger. Derartige Verbraucher können, z. B. zur Lastverstetigung, für begrenzte Zeiträume abgeschaltet werden.

\subsection{Zwischenfazit}

Noch haben sich tEnMS innerhalb der Prozessindustrie nicht vollumfänglich durchgesetzt. Es gibt allerdings Gründe, warum sich dies in der Zukunft ändern wird:

Zum einen wird ein Energiemanagementsystem und damit auch ein tEnMS in der ISO 50001 gefordert. Zum anderen wird im NA 140 die durchgängige Erfassung von Energiedaten als „Best Practice“ empfohlen und sie erscheint auch als Maßnahme zur Realisierung künftiger Energieeinsparungen sinnvoll. Besonders der Aspekt der Energiedatenerfassung wird dabei für zukünftige prozesstechnische Anlagen eine hohe Bedeutung haben. Es existieren allerdings auch vereinzelt Potenziale für das Lastmanagement, diese werden von den Autoren jedoch eher als gering eingeschätzt.

\section{Technische Energiemanagementsysteme}

Dieses Kapitel beschreibt den Aufbau und die möglichen Integrationsgrade von sich aktuell im Einsatz befindenden tEnMS [12, 15]. Wie bereits in der Einleitung erwähnt, dient ein tEnMS heute meist der Messung, Speicherung, Auswertung und Darstellung von Energiedaten. Gegebenenfalls wird die Realisierung eines Lastmanagements ergänzt.

\subsection{Separates technisches Energiemanagementsystem}

Ein typisches tEnMS lässt sich nach [16] in der Ebenenstruktur der Automatisierungstechnik prinzipiell auf der MESEbene verorten. Hier finden die Speicherung, die Auswertung und die Darstellung der Energiedaten statt. Außerdem werden hier ggf. die Schaltbefehle für das Lastmanagement generiert.

Ein tEnMS benötigt aber auch Systemteile auf den Ebenen unterhalb der MES-Ebene. Hier nehmen Messgeräte die Energiedaten auf. Die Vorverarbeitung, Skalierung und Bereitstellung der Energiedaten erfolgt durch eigens für das tEnMS realisierte SPS-Programme (Energie-SPS-Programme). Gegebenenfalls werden hier auch die Schaltbefehle aus dem Lastmanagement aufgenommen und koordiniert ausgeführt. Der Transport der Energiedaten erfolgt über ein industrielles Kommunikationssystem.

Die Teile des tEnMS unterhalb der MES-Ebene können in unterschiedlichem Maße in das vorhandene Automatisierungssystem integriert sein. Im einfachsten Fall existieren beide Systeme parallel und sind strikt getrennt aufgebaut. In diesem Fall wurden für die Aufnahme von Energiedaten eigene Messgeräte und Zähler (in der Abbildung 2 mit $M$ gekennzeichnet) installiert. Die Energie-SPS-Programme laufen auf eigens für das tEnMS installierten SPSen. Auch das Kommunikationssystem für die Energiedaten wird eigens für das tEnMS installiert. Ein Lastmanagement ist bei einem parallelen Aufbau von tEnMS und Automatisierungssystem nicht möglich, da hierzu eine Kopplung zwischen EnergieSPS-Programmen und Anwenderprogrammen auf den vorhandenen SPSen benötigt wird. Abbildung 2 zeigt ein parallel zur Automatisierungstechnik installiertes tEnMS.

\subsection{Integriertes technisches Energiemanagementsystem}

Es ist auch ein höherer Integrationsgrad von tEnMS in bestehende Automatisierungssysteme möglich. So können die 
Energie-SPS-Programme parallel zu den Anwenderprogrammen auf den vorhandenen SPSen betrieben werden. Zudem werden bereits vorhandene Kommunikationssysteme (Automatisierungsnetzwerke) für den Transport von Energiedaten genutzt. Abbildung 3 zeigt ein integriertes tEnMS.

In der Abbildung werden die integrierten tEnMS-Funktionen durch die blauen Kästen neben den E/A-Ebenen und Frequenzumrichtern dargestellt. Es wird das vorhandene Kommunikationssystem für die Energiedatenkommunikation genutzt.

\subsection{Zwischenfazit}

Der separate Aufbau eines Energiemanagementsystems bietet die Möglichkeit Energiedaten zu erfassen, ohne in die bestehende Struktur der Automatisierungslösung einzugreifen. Allerdings ist hierfür eine separate Infrastruktur aufzubauen und zu betreiben. Ein Energiemanagement (Verbrauchsbeeinflussung) ist nicht oder nur eingeschränkt möglich, da hier in der Regel eine Verknüpfung mit dem Automatisierungssystem erforderlich ist. Eine integrierte Lösung erlaubt die Nutzung der bestehenden Infrastruktur des Automatisierungssystems. Ein Lastmanagement wird einfacher möglich. Auch wenn kein Lastmanagement erfolgen soll, ist bei einer integrierten Lösung von einer Kostenersparnis durch Mitnutzung einer vorhandenen Infrastruktur auszugehen. Daher fokussiert der Rest des Beitrages auf eine integrierte Lösung.

\section{Das PROFlenergy-Energiemanagementprofil} Dieses Kapitel beschreibt das PROFlenergy-Energiemanagementprofil für PROFINET hinsichtlich Funktionsumfang und Anwendungsfällen. [8]

Die Kommunikation über das PROFlenergy-Energiemanagementprofil erfolgt ausschließlich kommandobasiert im Rahmen der azyklischen Kommunikation. Ein Kommando besteht aus einem Request des PROFINET-Controllers (üblicherweise der SPS) an ein Device und einer Response von dem Device zurück an den Controller. Welche Arten von Kommandos ein Device unterstützt, wird durch die sogenannte PE Entity Class festgelegt.

» Ein Device der PE Entity Class 1 unterstützt Kommandos zur Realisierung eines Standby-Managements. Das Device verfügt also über einen oder mehrere Energiesparmodi. Wenn das Device das Kommando zum Energiesparen bekommt, entscheidet es selbst anhand der übergebenen, erwarteten Pausenzeit, welchen Energiesparmodus es einnimmt. Devices der Entity Class 1 verfügen über keine Energiemessfunktionen, allerdings ist jedem Betriebs- und Energiesparmodus ein empirisch ermittelter konstanter Wert für einen Standardenergieverbrauch hinterlegt, die von dem Device abgerufen werden kann. So können auch Entity-Class-1-Devices in das Energiedatenerfassungsnetz eingebunden werden.

» Ein Device der PE Entity Class 2 bietet Energiemessfunktionen, verfügt also auch über integrierte Energiemessstellen. Gemessene Energiedaten können von den
Devices direkt abgerufen werden. Hierbei handelt es sich, neben den Energieverbräuchen, z. B. um Spannung, Strom, Schein-, Blind- und Wirkleistung, etc. Neben dem direkten Abruf von Energiemesswerten verfügen EntityClass-2-Devices seit der Version 1.3 des PROFlenergyEnergiemanagementprofils auch über Funktionen zur kontinuierlichen Energiemessung. Hierbei kann eine kontinuierliche Energiemessung per Kommando gestartet und beendet werden.

» Ein Device der PE Entity Class 3 verfügt sowohl über die Standby-Management-Funktionen eines Entity-Class1-Devices als auch über die Energiemessfunktionen eines Entity-Class-2-Devices.

In dem PROFlenergy-Whitepaper [20] sind vier Anwendungsfälle für das Energiemanagementprofil definiert:

1. Das Einsparen von Energie in kurzen, geplanten Stillständen von unter einer Stunde, wie z. B. Produktionspausen. In diesem Anwendungsfall sollen die Energiesparmaßnahmen nur die Verbraucher mit einer kurzen Wiederanlaufzeit betreffen, um die Anlagenverfügbarkeit nicht zu beeinträchtigen.

2. Das Einsparen von Energie in geplanten Stillstandzeiten von mehreren Stunden oder Tagen, wie z. B. über Nacht oder an Wochenenden. In diesem Anwendungsfall betreffen die Energiesparmaßnahmen auch Verbraucher mit längeren Anlaufzeiten.

3. Das Einsparen von Energie bei unvorhergesehenem Stillstand, z. B. bedingt durch Störungen in vor- oder nachgeschalteten Anlagen. Da die Dauer des Stillstandes hier zunächst nicht bekannt ist, tritt zunächst Anwendungsfall 1 in Kraft. Sollte sich dann herausstellen, dass der Stillstand länger andauern wird, kann in den Anwendungsfall 2 gewechselt werden.

4. Das Messen von Energieverbräuchen. Hier werden die Energiemesswerte von den PROFlenergy-fähigen Devices erfasst und dem Anwender in einer einheitlichen Form zur Verfügung gestellt.

5. Obwohl in dem PROFlenergy-Energiemanagementprofil selbst nur elektrische Größen als integrierte Energiemesswerte definiert sind, ist es für die Hersteller von PROFlenergy-fähigen Devices möglich, beliebige selbstdefinierte Energiedaten über PROFlenergy bereit zu stellen. So können auch nicht-elektrische, aber dennoch aus Energiesicht relevante Größen, wie z. B. Druck, Temperatur, etc., bereitgestellt werden.

\section{Nutzung von Energieinformationen intelli- genter Feldgeräte}

Dieser Abschnitt betrachtet, wie intelligente Feldgeräte zusätzlich zu den Messwerten Energieinformationen bereitstellen können. Dabei wird davon ausgegangen, dass 


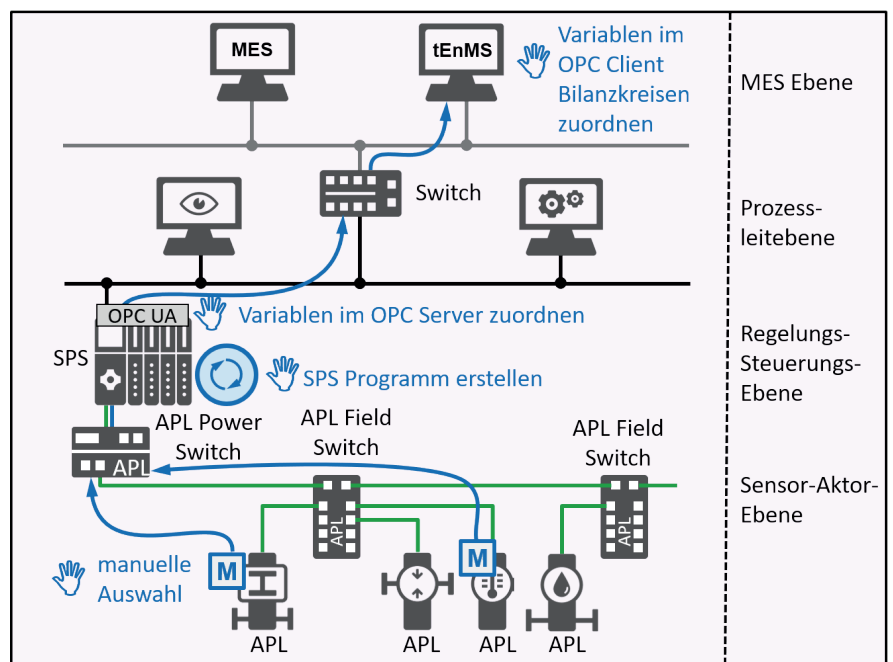

Abbildung 4: Manuelle Programmierung der Erfassung von Energiedaten aus intelligenten Messumformern.

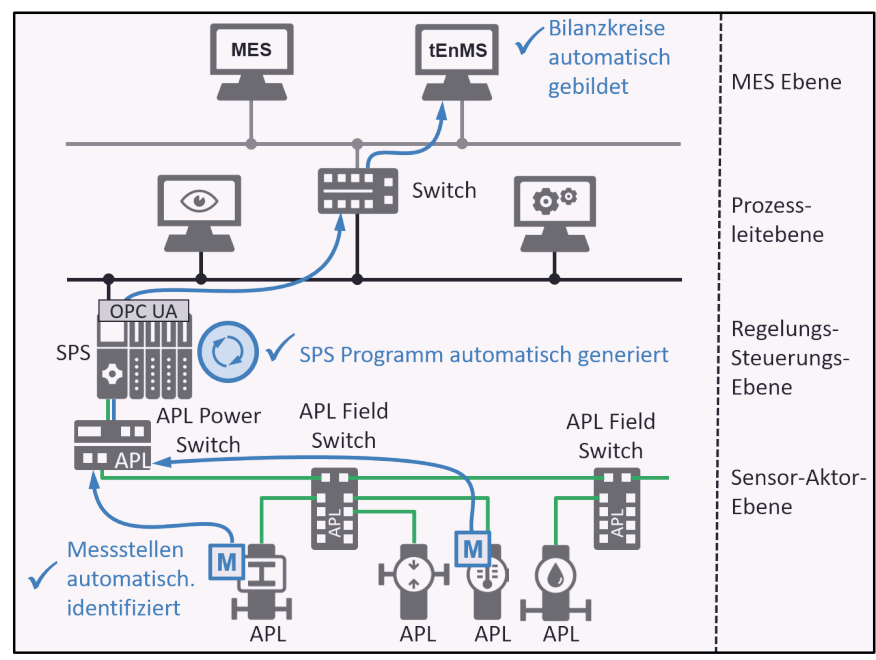

Abbildung 5: Automatisierte Programmierung der Erfassung von Energiedaten aus intelligenten Messumformern.

diese Messumformer künftig über einen Zweidraht-EthernetAnschluss nach IEEE 802.3cg (10SPE) verfügen. Damit könnten derartige Messumformer auch über Echtzeitprotokolle, wie z. B. PROFINET, kommunizieren und es steht ggf. auch das in Kapitel 3 beschriebene PROFlenergy-Energiemanagementprofil für die entsprechenden Sensoren und Aktoren zur Verfügung. Weitere Details zur dieser Technologie, die für die Prozessindustrie zurzeit unter dem Namen Ethernet APL spezifiziert wird, finden sich in [21].

\subsection{Manuelle Programmierung der Erfassung von Energie- daten aus Feldgeräten}

Die Erfassung von Energiedaten aus intelligenten Feldgeräten ist bereits heute auf Basis existierender Technologien möglich.

Abbildung 4 zeigt die Erfassung von Energiedaten unter Nutzung von Ethernet APL. Es wird davon ausgegangen, dass die Messumformer Messwerte liefern, die auch für Energiemanagementzwecke relevant sind. Bei der hier

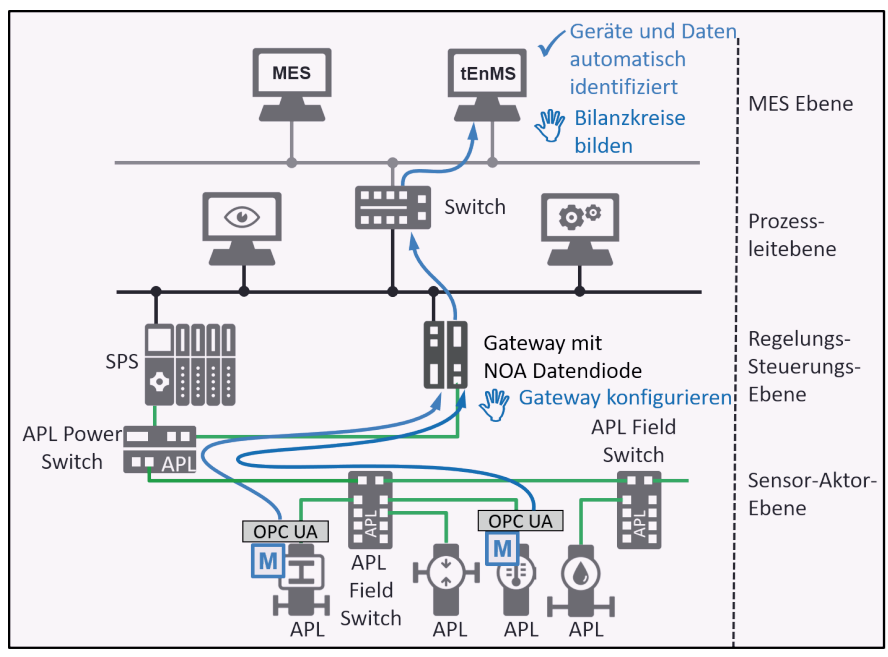

Abbildung 6: Erfassung von Energiedaten aus intelligenten Messumformern über integrierte OPC-UA-Server in den Messumformern.

beschriebenen manuellen Erfassung sind folgende Tätigkeiten auszuführen, um die Energiedaten in einem tEnMS verfügbar zu machen:

» Manuelle Auswahl der geeigneten Messgrößen.

» Manuelle Erstellung eines SPS-Programms, das die Daten erfasst und ggf. miteinander verrechnet.

》Bereitstellung der Daten in dem OPC-UA-Server der SPS. Eintragen der Variablen im Sever.

» Konfiguration des tEnMS Systems zur Erfassung der OPCUA-Variablen aus dem OPC-UA-Server der SPS

Hinweis: Manuelle Tätigkeiten sind in Abbildung 4 mit einem sy -Symbol gekennzeichnet. Ein vergleichbares Vorgehen ist heute auch schon unter Nutzung von z. B. PROFIBUS PA oder HART-Protokoll und ohne den Einsatz von Ethernet APL möglich. Es ist der hohe Anteil manueller Tätigkeiten bei diesem Vorgehen zu beachten.

\subsection{Automatisierte Programmierung der Erfassung von Energiedaten unter Nutzung von PROFlenergy}

In diesem Abschnitt wird nun davon ausgegangen, dass die Feldgeräte mit dem Energieprofil PROFlenergy gemäß PE Entity Class 2 ausgestattet sind. Die Gerätebeschreibungsdatei (GSD-Datei) des Feldgerätes enthält Informationen darüber, welche Energiemessgrößen das Gerät liefert.

Damit ist dem Engineering-Tool des Automatisierungssystems bekannt, welche Messumformer welche Energiedaten liefern und in welchem Format diese über das PROFlenergy-Profil in der SPS ankommen. Für die SPS kann eine automatische Codegenerierung und eine automatische Konfiguration des OPC-UA-Servers erfolgen, wie sie in $[17,18]$ beschrieben wurde. Da dem Engineering-Werkzeug auch die Anlagenstruktur bekannt ist, kann auch ein Vorschlag für die Zuordnung der Messtellen in Bilanzkreise automatisch erfolgen. 
Tabelle 1: Gegenüberstellung der Erfassungsmöglichkeiten.

\begin{tabular}{|c|c|c|c|}
\hline & $\begin{array}{l}\text { Manuelle Programmierung } \\
\text { (Kap. 4.1) }\end{array}$ & $\begin{array}{l}\text { Automatische Programm- } \\
\text { generierung in Verbindung } \\
\text { mit PROFlenergy } \\
\text { (Kap. 4.2) }\end{array}$ & $\begin{array}{l}\text { OPC UA-Server im } \\
\text { Feldgerät (Kap. 4.3) }\end{array}$ \\
\hline $\begin{array}{l}\text { Programmieraufwand für } \\
\text { Anwender, der Energiedaten } \\
\text { erfassen möchte }\end{array}$ & $\begin{array}{l}\text { Hoch } \\
\text { Geräteidentifikation, SPS- } \\
\text { Programmerstellung, Konfi- } \\
\text { guration OPC-UA-Server und } \\
\text { Bilanzkreisbildung erfolgen } \\
\text { manuell. }\end{array}$ & $\begin{array}{l}\text { Gering } \\
\text { Geräteidentifikation, SPS- } \\
\text { Programmerstellung, Konfi- } \\
\text { guration OPC-UA-Server und } \\
\text { Bilanzkreisbildung erfolgen } \\
\text { automatisiert. }\end{array}$ & $\begin{array}{l}\text { Mittel } \\
\text { Identifikation der Geräte } \\
\text { erfolgt automatisch. Konfigu- } \\
\text { ration NOA Datendiode und } \\
\text { Bilanzkreisbildung erfolgen } \\
\text { manuell. }\end{array}$ \\
\hline $\begin{array}{l}\text { Bereitstellung der Energie- } \\
\text { daten in einem standardi- } \\
\text { sierten Format }\end{array}$ & $\begin{array}{l}\text { Nutzerabhängig } \\
\text { Bei der manuellen Program- } \\
\text { mierung der Energiedaten } \\
\text { liegt es in der Hand des } \\
\text { Nutzers, inwieweit die Daten } \\
\text { standardisiert bereitgestellt } \\
\text { werden. Entsprechende } \\
\text { Formate müssen von den } \\
\text { Geräten unterstützt und } \\
\text { bereitgestellt werden. }\end{array}$ & $\begin{array}{l}\text { Hoch } \\
\text { Das PROFlenergy-Profil } \\
\text { definiert das Format der } \\
\text { Energiedaten. In diesem } \\
\text { Format werden die Daten } \\
\text { dem tEnMS übermittelt. }\end{array}$ & $\begin{array}{l}\text { Zur Zeit gering } \\
\text { Derzeit stehen noch keine } \\
\text { Energiedatenprofile für OPC } \\
\text { UA zur Verfügung. Es ist } \\
\text { jedoch davon auszugehen, } \\
\text { dass diese zu einem späteren } \\
\text { Zeitpunkt bereitgestellt wer- } \\
\text { den können. }\end{array}$ \\
\hline $\begin{array}{l}\text { Verfügbarkeit von Energie- } \\
\text { daten im Controller / SPS }\end{array}$ & $\begin{array}{l}\text { Ja } \\
\text { Die Daten werden im Control- } \\
\text { ler erfasst und dann von dort } \\
\text { an das tEnMS weitergeleitet. } \\
\text { Daher stehen alle Energieda- } \\
\text { ten auch lokal im Controller } \\
\text { zur Verfügung. }\end{array}$ & $\begin{array}{l}\text { Ja } \\
\text { Die Daten werden im Cont- } \\
\text { roller erfasst und dann von } \\
\text { dort an das tEnMS weiter- } \\
\text { geleitet. Daher stehen alle } \\
\text { Energiedaten auch lokal im } \\
\text { Controller zur Verfügung. }\end{array}$ & $\begin{array}{l}\text { Nein } \\
\text { Die Energiedaten gehen direkt } \\
\text { vom Messumformer zum } \\
\text { Gateway. Um die Daten im } \\
\text { Controller verfügbar zu ma- } \\
\text { chen, müssten diese zusätz- } \\
\text { lich, z. B. als zyklische Daten, } \\
\text { an den Controller übertragen } \\
\text { werden. }\end{array}$ \\
\hline $\begin{array}{l}\text { Implementierungsaufwand } \\
\text { im Feldgerät }\end{array}$ & $\begin{array}{l}\text { Keiner } \\
\text { Diese Lösung arbeitet nur mit } \\
\text { der bereits im Gerät vorhan- } \\
\text { denen Funktionalität. }\end{array}$ & $\begin{array}{l}\text { Mittel } \\
\text { Im Gerät ist zusätzlich das } \\
\text { PROFlenergy-Profil zu } \\
\text { hinterlegen. Im Kontext von } \\
\text { [17] haben die Autoren ein } \\
\text { solches Profil selbst imple- } \\
\text { mentiert und schätzen den } \\
\text { Aufwand auf Grund eigener } \\
\text { Erfahrung als mittel ein. }\end{array}$ & $\begin{array}{l}\text { Mittel } \\
\text { Im Feldgerät ist ein zusätz- } \\
\text { licher OPC-UA-Server zu } \\
\text { implementieren. }\end{array}$ \\
\hline $\begin{array}{l}\text { Zusätzlicher Ressourcen- } \\
\text { verbrauch im Feldgerät } \\
\text { (Speicher, Rechenleistung) }\end{array}$ & $\begin{array}{l}\text { Keiner } \\
\text { Diese Lösung arbeitet nur mit } \\
\text { der bereits im Gerät vorhan- } \\
\text { denen Funktionalität. }\end{array}$ & $\begin{array}{l}\text { Gering } \\
\text { Das PROFlenergy-Profil } \\
\text { arbeitet über azyklische } \\
\text { Kommunikation und die } \\
\text { Energiedaten werden nur } \\
\text { in größeren Zeitabständen } \\
\text { abgerufen. }\end{array}$ & $\begin{array}{l}\text { Mittel } \\
\text { Der „Fußabdruck“ eines } \\
\text { Embedded-OPC-UA-Servers } \\
\text { ist größer, als der des PROFI- } \\
\text { energy-Profils, da hier parallel } \\
\text { zu PROFINET ein komplettes } \\
\text { zweites Kommunikations- } \\
\text { protokoll implementiert wird. }\end{array}$ \\
\hline $\begin{array}{l}\text { Ableitung von Schaltbefehlen } \\
\text { aus den Energiedaten }\end{array}$ & Direkt in der SPS möglich & Direkt in der SPS möglich & $\begin{array}{l}\text { Über tEnMS via Befehl an die } \\
\text { SPS möglich }\end{array}$ \\
\hline
\end{tabular}


Für die Erfassung von Energiedaten über das PROFlenergyProfil fallen somit die folgenden Arbeitsschritte an:

» Automatische Identifikation der Geräte, die Energiedaten liefern können über die GSD-Dateien der Geräte und den Import in das Engineering-Tool.

» Automatische Erzeugung des Programmcodes für die SPS, um die Energiedaten aus den Sensoren zu erfassen und im OPC-UA-Server der SPS bereitzustellen, wie in $[17,18]$ beschrieben

» Automatische Konfiguration des OPC UA Servers in der SPS, wie in $[17,18]$ beschrieben.

» Automatisierte Erzeugung von Bilanzkreisen im tEnMS wie in [17, 18,] beschrieben, da das Engineering-Werkzeug über Strukturinformationen der Automatisierungsanlage verfügt. Hierfür kann auch ein zusätzlicher Aggregationsserver (in der Abbildung nicht dargestellt) verwendet werden.

Da über das Energiemanagementprofil die Energiedaten in einem standardisierten Formal bereitgestellt werden, kann eine automatische Erzeugung der Software zur Weiterverarbeitung der Daten in den überlagerten Systemen erfolgen.

\subsection{Erfassung der Energiedaten über OPC UA Server in den Feldgeräten}

Die bisher beschriebenen Konzepte gehen davon aus, dass die Energiedaten zunächst an eine SPS übertragen werden und dort dann in einem OPC UA Server für die übergeordneten Ebenen bereitgestellt werden. Dieser Abschnitt geht nun davon aus, dass die Feldgeräte selbst über einen integrierten OPC UA Server [22] verfügen.

Abbildung 6 zeigt eine mögliche Struktur für eine Erfassung der Energiedaten über integrierte OPC UA Server in den Messumformern. Die Daten werden über ein Gateway mit NOA-Datendiode [19] für die MES-Ebene bereitgestellt. Durch ein Browsen des OPC-UA-Adressraums können alle Messumformer, die Energiedaten bereitstellen, identifiziert werden. Zurzeit steht jedoch noch kein standardisiertes Energieprofil für Messumformer über OPC UA zur Verfügung, so dass hier von einem heterogenen Informationsbestand auszugehen ist. Für die Erfassung der Energiedaten sind die folgenden Arbeitsschritte erforderlich:

" Manuelle Konfiguration der NOA-Datendiode, um ein Browsen der Feldgeräte durch das tEnMS zu ermöglichen.
» Dadurch automatischer Aufbau einer Datenbasis mit Geräte- und Dateninformation, allerdings in einem nicht standardisierten Format.

» Manuelle Konfiguration der Bilanzkreise.

Durch die gewählte Architektur haben die SPSen/Prozesscontroller keinen Zugriff auf die Energiedaten. Hierfür müssten die SPSen einen OPC Client implementieren oder die Energiedaten müssen zusätzlich auch als Prozessdaten zyklisch kommuniziert werden.

\section{Diskussion und Ableitung von Handlungs- empfehlungen}

In diesem Kapitel werden die drei im vorangehenden Kapitel beschriebenen Alternativen zur Erfassung von Energiedaten verglichen und bewertet. Im Anschluss daran werden Handlungsempfehlungen abgegeben.

Die Gegenüberstellung in Tabelle 1 zeigt, dass alle drei Lösungen Vor- und Nachteile aufweisen. Die manuelle Lösung erfordert den höchsten Arbeitsaufwand, kann aber schon heute auf Basis bestehender Technologien (z. B. über PROFIBUS PA oder HART) eingesetzt werden. Ethernet APL oder intelligente Messumformer sind für diese Lösung nicht zwangsläufig erforderlich. Das Konzept unter Einsatz von PROFlenergy hat den Vorteil, dass die Energiedaten in einem standardisierten Format bereitgestellt werden und dass dadurch eine automatische Codegenerierung und eine automatische Strukturierung der Energiedaten möglich ist. OPC UA ermöglicht einen direkten Zugriff auf die Energiedaten, ohne dass diese an den Controller oder die SPS kommuniziert werden müssen. Allerdings stehen die Energiedaten in diesem Fall nicht in der SPS zur Verfügung, um daraus z. B. direkte Schalthandlungen abzuleiten.

\section{Fazit}

In diesem Beitrag wurde gezeigt, dass PROFlenergy gute Eigenschaften für eine Energiedatenerfassung im Rahmen von tEnMS in Anlagen der Prozessindustrie aufweist. Der entscheidende Punkt hierbei ist, dass PROFlenergy eine standardisierte, hersteller- und gerätetypunabhängige Schnittstelle für Energiedaten bereitstellt und damit eine automatisierte Generierung von Programmcode in der SPS ermöglicht. Die Bereitstellung von Energiedaten über OPC UA wird dann eine Alternative werden, wenn OPC UA standardisierte Energieprofile bereitstellt. Allerdings ist davon auszugehen, dass der Ressourcenbedarf im Feldgerät für den zusätzlichen OPC UA Stack höher sein wird als beim PROFlenergy-Profil. 


\section{Referenzen}

[1] Bundesministerium für Umwelt, Naturschutz und nukleare Sicherheit (BMU). (2019). Klimaschutz in Zahlen - Fakten, Trends und Impulse deutscher Klimapolitik. Abgerufen von: https://www.bmu.de/fileadmin/ Daten_BMU/Pools/Broschueren/klimaschutz_zahlen_2019_broschuere_bf.pdf

[2] Müller, E., Engelmann, J., Löffler, T., Jörg, S. (2009). Energieeffiziente Fabriken planen und betreiben. Springer-Verlag.

[3] Bundesministerium für Umwelt, Naturschutz und nukleare Sicherheit (BMU). (2020). Stromverbrauch: Entwicklung und Maßnahmen. Abgerufen von: https://www.umweltbundesamt.de/daten/energie/stromverbrauch

[4] Statista. (2020). Energieverbrauch des Verarbeitenden Gewerbes in Deutschland nach ausgewählten Sektoren im Jahr 2018. Abgerufen von: https://de.statista.com/statistik/daten/studie/432596/umfrage/energieverbrauch-im-verarbeitenden-gewerbe-in-deutschland-nach-sektor/

[5] DIN EN ISO 50001. (2011). Energiemanagementsysteme - Anforderung mit Anleitung zur Anwendung. DIN: www.beuth.de

[6] VDI 4602 Blatt 1. (2007). Energiemanagement Begriffe. VDI: www.vdi.de

[7] Rossiter, A. P., Jones, B. P. (Eds.). (2015). Energy management and efficiency for the process industries. John Wiley \& Sons.

[8] PROFIBUS Nutzerorganisation e. V. (PNO). (2019). Common Application Profile PROFlenergy: Technical Specification for PROFINET Version V1.3. 2019. Abgerufen von: https://www.profibus.com/download/profienergy/

[9] Schlechtendahl, J. (2012). Whitepaper SERCOS Energy. Abgerufen von: https://dc-us.resource.bosch.com/media/en/general_use/products/engineering/sercosiii/files_1/sercos_energy_whitepaper_en.pdf

[10] Zhu, F. (2014). Energy and process optimization for the process industries. Wiley, Hoboken, N.J., 2014.

[11] Awuah-Offei, K. (2018). Energy Efficiency in the Minerals Industry. Springer, Cham.

[12] Güldner, F., Menze, T. (2014). ARC Advisory Group-Energy Management Online Survey The Findings. ARC Advisory Group.
[13] NA 140. (2012). Vorgehensweise zur Steigerung der Energieeffizienz in chemischen Anlagen Beitrag der Automatisierungstechnik. NAMUR: www. namur.net

[14] Würger, A. Niemann, K.-H. (2018). Einfluss von Industrie 4.0 auf die Anwendbarkeit von Lastmanagement in der industriellen Produktion. In Tagungsband AALE 2018, Köln, 01. - 02.03.2018, S. 55-66.

[15] Niemann, K. H. (2011). Bestandsaufnahme von Energiemanagementfunktionen in Automatisierungssystemen: Studie für den Zentralverband Elektrotechnik- und Elektronikindustrie (ZVEI). Abgerufen von: https://nbn-resolving.org/urn:nbn:de:bsz:960-opus4-11393

[16] VDI 5600 Blatt 6. (2016). Fertigungsmanagementsysteme (Manufacturing Execution Systems - MES): Energiemanagement mit MES. VDI: www.vdi.de

[17] Würger, A., Niemann, K. H., Fay, A., Gienke, M., \& Paulick, M. (2019). Integriertes Anlagenengineering zur Erhöhung der Energieeffizienz. atp magazin, 61(11-12), 70-77.

[18] Würger, A. (2020). Automatisierte Generierung von Energiemanagementfunktionen auf der Basis des PROFINET-Energieprofils. Dissertation. Helmut-Schmidt-Universität, 2020. DOI: 10.24405/9095.

[19] NE 175. (2020). NAMUR Open Architecture - NOA Konzept. NAMUR: www. namur.net

[20] PROFIBUS Nutzerorganisation e. V. (PNO). (2010). PI White Paper: The PROFlenergy Profile: Increasing the Energy Efficiency of Automation Systems using Smart Energy Management over PROFINET. Abgerufen von: https:// www.profibus.com/download/pi-white-paper-the-profienergy-profile/

[21] PROFIBUS und PROFINET International, ODVA Inc., OPC-Foundation, FieldComm Group. (o.J.). Ethernet - To the Field. Abgerufen von: https://www.profibus.com/index.php?elD=dumpFile\&t=f\&f=107608\&token=97abc376e3e83e010df1902d93deba94d1a30d22.

[22] Lange, J., Iwanitz, F. Burke, T. J. (2010). OPC: Von Data Access bis Unified Architecture, 4. Aufl. VDE-Verlag, Berlin, Offenbach, 2010.

AUTOREN

Prof. Dr.-Ing. Karl-Heinz Niemann (geb. 1959) vertritt seit 2005 die Lehrgebiete Prozessinformatik und Automatisierungstechnik an der Hochschule Hannover. Von 2002 bis 2005 war er an der Fachhochschule Nordostniedersachsen für das Lehrgebiet Prozessdatenverarbeitung verantwortlich. Davor war er in leitender Stellung in der Entwicklung von Prozessleitsystemen tätig, unter anderem bei ABB, Elsag Bailey und Hartmann \& Braun.

\author{
Prof. Dr.-Ing. Karl-Heinz Niemann \\ Hochschule Hannover \\ Fakultät I - Elektro- und Informationstechnik \\ Postfach 920261 \\ 30441 Hannover \\ (c) +49 (0) 51192961264 \\ @ karl-heinz.niemann@hs-hannover.de
}

Dr.-Ing. Andreas Würger (geb. 1989) war von 2017 bis April 2020 wissenschaftlicher Mitarbeiter im Forschungsprojekt „integriertes Anlagenengineering zur Erhöhung der Energieeffizienz IAE4" an der Hochschule Hannover. Davor studierte er an der Hochschule Hannover den Bachelorstudiengang Elektro- und Informationstechnik sowie den Masterstudiengang Sensor- und Automatisierungstechnik. Vor und während seines Studiums war er bei der MARS GmbH in Verden beschäftigt. Aktuell ist er als Applikationsingenieur bei der Phoenix Contact Electronics $\mathrm{GmbH}$ beschäftigt.
Dr.-Ing. Andreas Würger
Phoenix Contact Electronics $\mathrm{GmbH}$
Dringenauer Str. 30
31812 Bad Pyrmont
@ awuerger@phoenixcontact.com 